\title{
Adaptive Design: A Review of the Technical, Statistical, and Regulatory Aspects of Implementation in a Clinical Trial
}

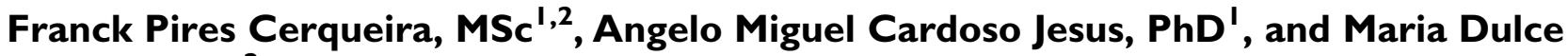 \\ Cotrim, $\mathbf{P h D}^{2}$ \\ 'Polytechnic Institute of Porto School of Health, Department of Pharmacy Porto, Portugal \\ ${ }^{2}$ Pharmacy Faculty of the University of Coimbra, Department of Pharmacology Coimbra, Portugal
}

\begin{abstract}
Background: In an adaptive trial, the researcher may have the option of responding to interim safety and efficacy data in a number of ways, including narrowing the study focus or increasing the number of subjects, balancing treatment allocation or different forms of randomization based on responses of subjects prior to treatment. This research aims at compiling the technical, statistical, and regulatory implications of the employment of adaptive design in a clinical trial. Methods: Review of adaptive design clinical trials in Medline, PubMed, EU Clinical Trials Register, and ClinicalTrials.gov. Phase I and seamless phase I/II trials were excluded. We selected variables extracted from trials that included basic study characteristics, adaptive design features, size and use of independent data-monitoring committees (DMCs), and blinded interim analysis. Results: The research retrieved 336 results, from which 78 were selected for analysis. Sixty-seven were published articles, and II were guidelines, papers, and regulatory bills. The most prevalent type of adaptation was the seamless phase II/III design $23.1 \%$, followed by adaptive dose progression $19.2 \%$, pick the winner / drop the loser $16.7 \%$, sample size re-estimation $10.3 \%$, change in the study objective $9.0 \%$, adaptive sequential design $9.0 \%$, adaptive randomization $6.4 \%$, biomarker adaptive design $3.8 \%$, and endpoint adaptation $2.6 \%$. Discussion: It is possible to infer that the use of Adaptive Design is an ethical and scientific advantage when properly planned and applied, since it increases the flexibility of the trial, shortens the overall clinical investigation time of a drug, and reduces the risk of patient exposure to adverse effects related to the experimental drug. Its greater methodologic and analytic complexity requires an adequate statistical methodology. Conclusions: The application of "adaptive clinical designs" for phase II/III studies appear to have been limited to trials with a small number of study centers, with smaller extensions of time and to experimental drugs with more immediate clinical effects that are amenable to risk/benefit decisions based on interim analyses. According to the reviewed studies, simple adaptive trial designs - such as early study terminations due to futility and sample size re-estimation-are becoming widely adopted throughout the pharmaceutical industry, especially in phase II and III studies. The pharmaceutical industry and contract research organizations (CROs) are implementing simple adaptations more frequently and the more complex adaptations-biomarker adaptive design, endpoint adaptation—are more sporadic.
\end{abstract}

\section{Keywords}

clinical trials, adaptive design, technical challenges, statistical limitations

\section{Introduction}

In a conventional clinical trial (CT), the parameters inherent to the study are defined in the protocol and remain unchanged during the study. Considering several factors of uncertainty and bias that may arise before the start of the CT (eg, target population change, duration of therapy, active control), a sequential design may present limitations due to erroneous assumptions formulated in the planning phase, even if the treatment under study is effective. ${ }^{1}$
The vast majority of protocols undergo changes during the conduct of the trial. Minor alterations are introduced through addenda to the initial protocol because they do not interfere in the scientific and statistical validity of the initial hypothesis, objective or endpoints. Changes that affect the validity of these factors (major changes), as well as factors that affect human subject rights (ability of withdrawal from the trial, protection of 
sensitive personal information, and ensured informed consent) need validation by request of authorization to the regulatory authorities and ethics committee. ${ }^{2}$

A major difference in an adaptive design (AD) context is that these changes are anticipated and projected prospectively in the protocol and/or statistical analysis plan (SAP) at the time of its elaboration. Another characteristic inherent to this type of design is the fact that the review and weighting of the results of the test are done through internal data from the study, acquired during the conduct of the study and not from information from external sources, as results of other tests with similar features. ${ }^{3}$

Despite the adaptations introduced in conducting the study, it is imperative to safeguard the scientific validity of the test by ensuring valid statistical inferences that will translate into correct results. To achieve this, operational biases need to be minimized and it may be necessary to introduce statistical adjustment (eg, $P$ value adjustment or redefinition of margins of error), which will assist in controlling type I error.

In an adaptive trial, the scientific and statistical validity is preserved mainly by the maintenance of blinding, but also by the prospective nature of the introduced adaptations. ${ }^{4}$

\section{Types of Adaptive Design}

The variety of possible modifications in CT design described prospectively in the protocol (or separately, in a statistical analysis plan [SAP]) is vast. Examples include modifications to the following ${ }^{5-7}$ :

- Criteria for inclusion in the trial (either for subsequent inclusion in the study or for selection of a subgroup in a patient population)

- Randomization procedure

- Treatment regimens of different study groups (eg, dose, dosage, duration of treatment)

- Size of the study population (including cases of early completion)

- Concomitant treatment used

- Schedule of events (eg, number of interim analysis, timing of last evaluation of the last patient and duration of patient participation in the $\mathrm{CT}$ )

- Primary endpoint (eg, selection of outcome evaluation, assessment point determination)

- Selection or addition of surrogate endpoints

- Endpoint analysis (eg, statistical methodology, control of the type I error)

Based on the above modifications, the adaptations considered in CTs include (1) adaptive randomization, (2) adaptive sequential design, (3) sample size re-estimation, (4) "pick the winner," (5) adaptive dose progression, (6) phase II/III "seamless," (7) biomarker adaptive design, and (8) adaptation of trial hypothesis (Table 1).

\section{Institutional Guidelines on Implementing an Adaptive Design}

Two guidance documents on $\mathrm{AD}$ have been drawn up at least in part as a result of the pharmaceutical industry's advocacy efforts: the European Medicines Agency (EMA Reflection Paper 2007) ${ }^{8}$ and the Food and Drug Administration (FDA Draft Guidance 2010). ${ }^{9}$ The first is a relatively brief document, focusing almost exclusively on confirmatory studies, which neither encourages nor rules out the use of $\mathrm{AD}$ from a regulatory standpoint.

The FDA guidelines are considerably more detailed, covering both exploratory and confirmatory studies (especially on the latter), and elucidating not only potential constraints on the use of $\mathrm{AD}$ but also recommendations on how to overcome them in the development of a new drug or medical device. ${ }^{9}$

\section{EMA: reflection paper on adaptive design}

The European Medicines Agency (EMA) Reflection Paper has a pioneering role in the regulatory orientation of $\mathrm{AD}$ and has been published at a time of intense discussion of various aspects such as methodology, implementation, and regulatory validation. The EMA document is not as detailed as the FDA document and has a more limited scope, but it emphasizes some regulatory aspects about $\mathrm{AD}$ that are only tangentially discussed in the FDA document, making it a useful adjunct to the latter.

The EMA reflection paper focuses almost exclusively on confirmatory studies. The general guideline of the document is to validate adaptive design and its potential usefulness, but with clear attention to its adequate implementation in the practice of clinical research. Comparatively, it is less encouraging about AD than the FDA document but does not neglect its use when properly planned, conducted, and analyzed. In its initial considerations, the EMA document recognizes that the $\mathrm{AD}$ has the potential to shorten the development time of new drugs and to allocate resources more efficiently without compromising scientific and regulatory criteria. ${ }^{8}$

The EMA reflection paper is less didactic than the FDA guidelines, with no attempt to classify ADs (the FDA proposes to rank "well-understood" and "less well-understood"). A more formal definition of $\mathrm{AD}$ is included on the last page of the document and illustrates the limited view of the document: "a study is called 'adaptive' if the statistical methodology allows the modification of a design element... in an interim analysis with complete control of type I error." "From this definition, it becomes clear that the main concern in the document about the validity of an $\mathrm{AD}$ is the control of the type I error rate in the presence of possible adaptations.

In line with the FDA document, EMA's review formalizes that any step in implementing an AD must be preplanned, adequately justified in the context of the trial developed and kept to the minimum necessary. Tight control of type I error rate is indicated as a prerequisite for regulatory authorization of $\mathrm{AD}$ as well as use of appropriate statistical methods for 
determination of therapeutic effect $(P$ value and margin of error) is also required..$^{9}$

FDA: guidelines on implementation of adaptive design

The overall tone of the FDA guidelines is encouraging for the application of $\mathrm{AD}$, but with caution: the FDA recognizes $\mathrm{AD}$ as having potential to improve the efficiency and success rate of the development of a new drug but raises some questions about its application in confirmatory tests. It also recognizes that the greatest appeal of ADs is to allow preplanned interim corrections throughout the trial, review study design assumptions, and readjust endpoints based on the data obtained. Two main points of attention are expressed at the beginning and in the course of the guidelines: the possible inflation of the type I error rate and the increase in operational bias, which may compromise the scientific integrity of the study and the validity/ interpretation of the final results. ${ }^{1}$

There are statistical methods to adequately control type I error for a wide variety of ADs based on unblinded data (there is less influence of type I error when adaptations are made on blinded data), but it is emphasized that the sponsor is in order to demonstrate analytically that statistical analysis methods will control the type I error rate. ${ }^{9}$

Another point of discussion related to $\mathrm{AD}$ exposed by the FDA is more difficult to determine and control: the potential operational bias due to leakage of unblinded data over the course of the trial. Changes in the population of subjects after adaptation without blinding are cited examples of operational bias associated with $\mathrm{AD}$. Of course, such changes may also occur when no adaptations are introduced in the trial because they may result, for example, from the start of recruitment at other centers at later points in the study. ${ }^{9}$

FDA guidelines explicitly support the use of $\mathrm{AD}$ in the context of exploratory trials, attesting that they provide a model for additional dose-response, subgroup-based knowledge, and have the potential to promote substantial gains in clinical research efficiency.

\section{Objectives}

The use of adaptive design in clinical trials has been debated for several years and there is already regulatory documentation on its application (FDA Guidelines and EMA Discussion Paper) as well as medicines developed using these methods in several countries.

Considering the amount of information available, the limited employment of adaptive design methodology in clinical trials and the limited amount of published results in this area, it is justified the constitution of this research as an updated body of work to

- contextualize adaptive design in regulatory and terms of applicability in a clinical trial;

- analyze and compare European and American (US) guidelines;
- describe the implementation of adaptive design in a clinical trial in terms of planning, subject selection, interaction with regulators, and monitoring;

- describe the analysis and statistical protocol in terms of simulation, modeling, and bias;

- to present advantages and limitations of the application of adaptive design in clinical trials; and

- assess the future applications of adaptive design in clinical trials.

\section{Materials and Methods}

\section{Search Strategy and Eligibility Criteria}

Considering the given objective, the present research is a literature review. In order to build the documental body, several databases of specialized literature were used: ClinicalTrials.gov, PubMed, Medline, EU Clinical Trials Register, using the terms Adaptive Clinical Trials, Adaptive Design, and Adaptive Trials, with articles and publications published between 2006 and 2017.

We excluded nonhuman studies, systematic reviews, metaanalysis, editorials, and opinion articles. Incomplete trials still in progress were excluded to avoid misrepresenting trials, which can sometimes change format while underway. Only trials with adults were considered ( $>18$ years old).

Phase I and seamless phase I/II trials we excluded because such exploratory trials usually have low impact on regulatory approval and/or clinical uptake of treatments.

\section{Results}

The research retrieved 336 results, from which 78 were selected for analysis. Of those, 67 were published articles and 11 were guidelines, papers, and regulatory bills (Figure 1).

The most prevalent type of adaptation was the seamless phase II/III design, 18/78 (23.1\%); followed by adaptive dose progression, $15 / 78$ (19.2\%); pick the winner / drop the loser, 13/78 (16.7\%); sample size re-estimation, 8/78 (10.3\%); change in the study objective, 7/78 $(9.0 \%)$; adaptive sequential design, $7 / 78$ (9.0\%); adaptive randomization, 5/78 (6.4\%); biomarker adaptive design, $3 / 78$ (3.8\%); and endpoint adaptation, $2 / 78(2.6 \%)$ (Figure 2). Numerous trials incorporated more than 1 adaptation.

The selected trials were submitted under the scope of FDA and EMA. Overall, 56/78 (71.8\%) were approved without the need of additional information submission or changes in study protocols. Sixteen of $78(20.5 \%)$ were required to be resubmitted to establish changes in study design or provide additional information regarding scientific validity of the study according with the ADs applied. Three of 78 (3.84\%) had an approval limited to FDA regulations of AD (EMA has a more conservative stance on $\mathrm{AD}$ and does not cover less used types of AD: some types of nonvalidated biomarkers, adaptation of endpoints, and change in study hypothesis) (Table 2). 


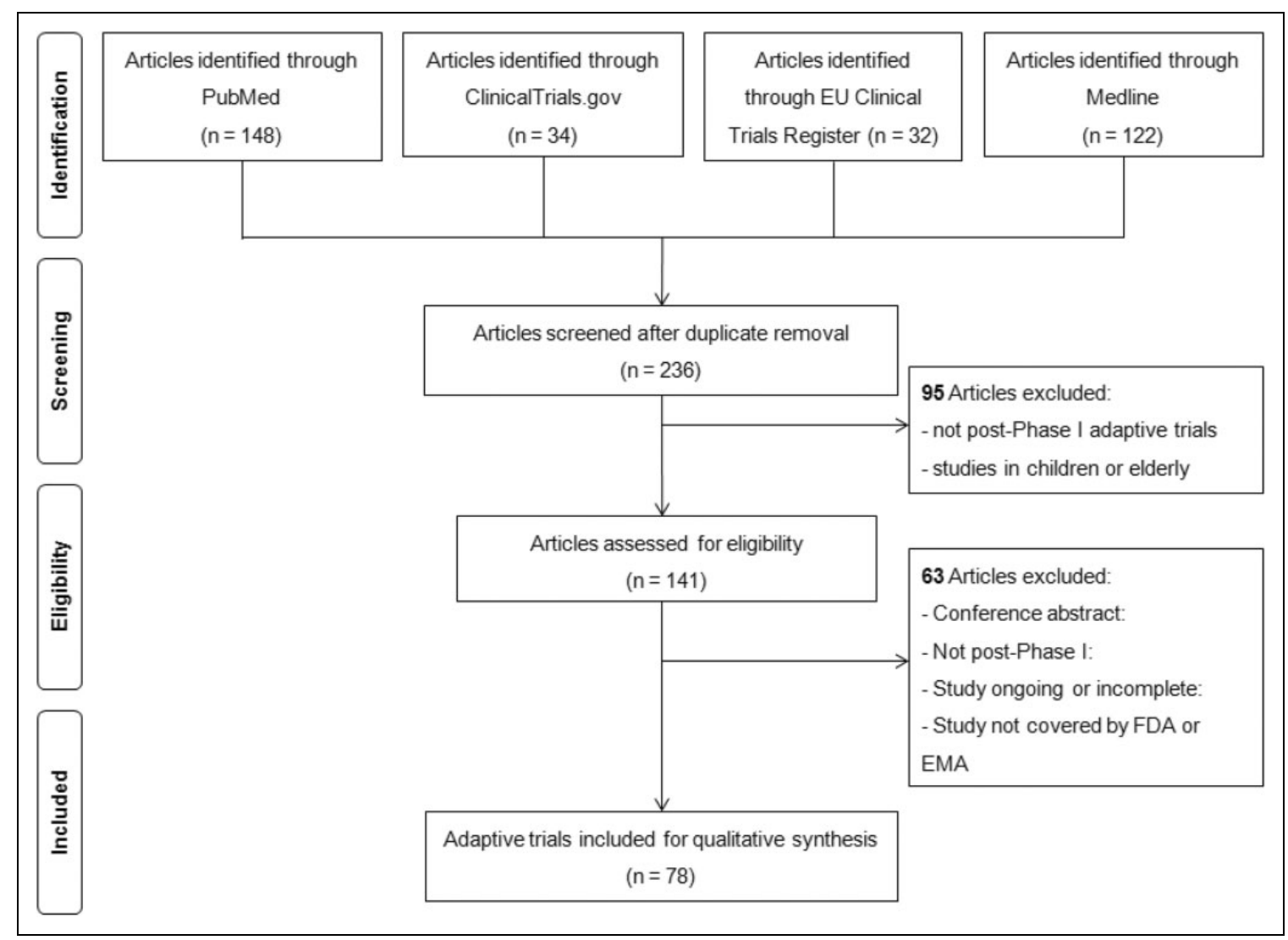

Figure I. Flow diagram derived from preferred reporting items for systematic reviews and meta-analyses (PRISMA). This diagram reports results of a search of published adaptive design clinical trials in PubMed on January 17, 2018, ClinicalTrials.gov on January I7, 20I8, EU Clinical Trials Register on February 23, 2018, and Medline on April 13, 2018.

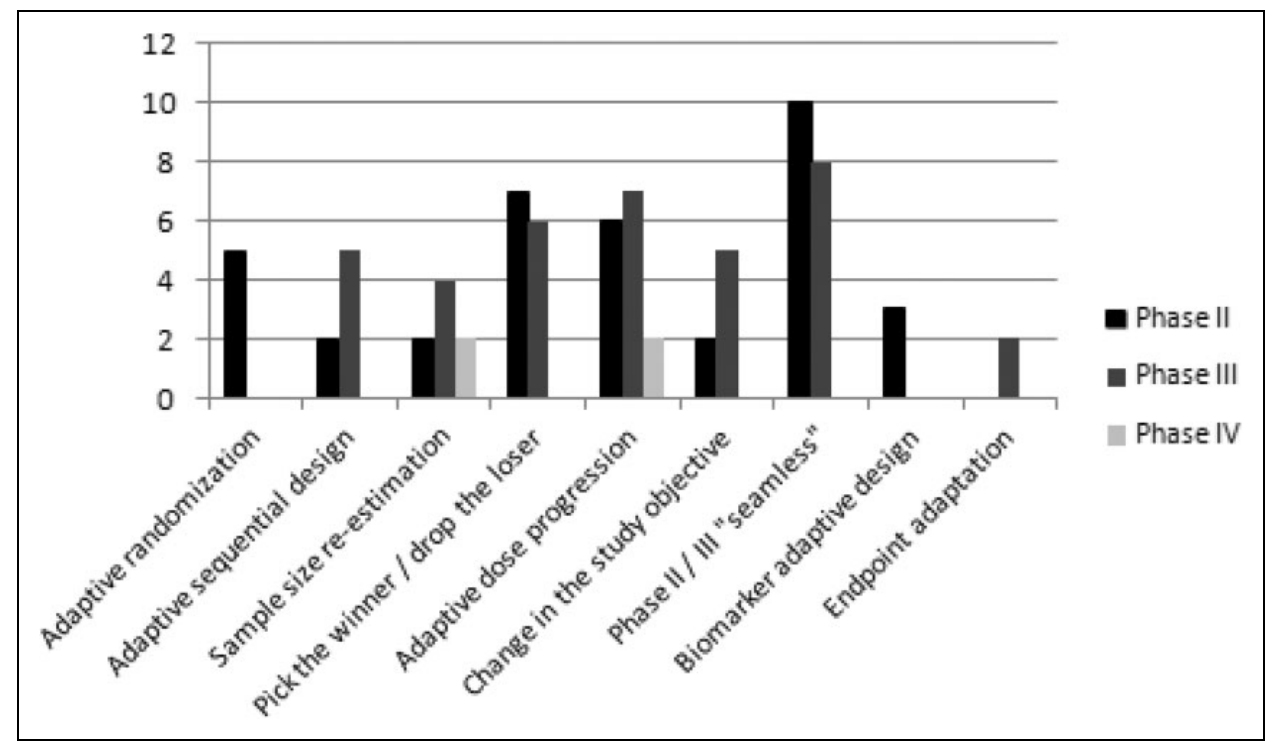

Figure 2. Distribution of adaptive design type in surveyed trials per phase $(n=78)$.

ADs more frequently used are pick the winner / drop the loser, phase II/III seamless, adaptive dose progression, and adaptive sequential design, thus having a better regulatory clearance rate. Designs like endpoint adaptation, change in study objective, and use of $\mathrm{AD}$ with biomarker are still in a more precocious stage of development that leads the 
Table I. Definition of Types of Adaptive Designs. ${ }^{\text {a }}$

\begin{tabular}{|c|c|}
\hline Type of Adaptive Design & Definition \\
\hline Adaptive sequential design & $\begin{array}{l}\text { Results are analyzed at interim analyses, with prespecified options of making adaptations such as sample size re- } \\
\text { estimation, modification/deletion/addition of treatment arms, changing study endpoints, modifying dose and/or } \\
\text { treatment duration, or adapting randomization schedules. }\end{array}$ \\
\hline Sample size re-estimation & Flexible sample size adjustment or re-estimation based on interim analysis of accumulating data. \\
\hline $\begin{array}{l}\text { Pick the winner / drop the } \\
\text { loser }\end{array}$ & $\begin{array}{l}\text { Possibility of dropping the inferior treatment group(s), modifying treatment arms and/or adding additional arms } \\
\text { based on the review of accumulating data at interim analysis. }\end{array}$ \\
\hline $\begin{array}{l}\text { Adaptive dose } \\
\text { progression }\end{array}$ & $\begin{array}{l}\text { Allocation of patients to multiple different treatment doses and patient responses are assessed at interim analyses. } \\
\text { Trial design is then adapted to allocate more patients to the treatment doses of interest, reducing allocation of } \\
\text { patients to doses that appear noneffective. }\end{array}$ \\
\hline Biomarker adaptive design & $\begin{array}{l}\text { Adaptations to the trial design based on interim analysis of the treatment responses of biomarkers, such as genomic } \\
\text { markers. This design can be used to select patient populations for subsequent trials, identify the natural course of } \\
\text { a disease, achieve early detection of a disease, and/or help in developing personalized medicine. }\end{array}$ \\
\hline $\begin{array}{l}\text { Adaptation of trial } \\
\text { hypothesis }\end{array}$ & $\begin{array}{l}\text { Trial hypotheses are adapted in response to interim analysis results. They may involve a preplanned shift from a } \\
\text { single hypothesis to multiple hypotheses or preplanned switching between the primary and secondary study } \\
\text { endpoints. }\end{array}$ \\
\hline
\end{tabular}

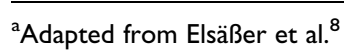

Table 2. Submission Success of Adaptive Design Trials $(n=78)$.

\begin{tabular}{lrrll}
\hline Type of Adaptive Design & Cleared & & Cleared With Resubmission $^{\mathrm{a}}$ \\
\hline Adaptive randomization & 3 & 2 & Request of independent statistical analysis plan \\
Adaptive sequential design & 5 & 2 & Justification of timing and frequency of interim analyses \\
Sample size re-estimation & 6 & 2 & Control of inflation of type I error \\
Pick the winner / drop the loser & 12 & I & Definition of efficacy and safety targets for futility \\
Adaptive dose progression & 12 & 3 & Adjustment of therapeutic dose regimen \\
Phase II/III "seamless" & 16 & 2 & Control of inflation of type I error due to inflation of sample size \\
Biomarker adaptive design & I & I & Request of further information for biomarker validation \\
Endpoint adaptation & & I Redefinition of rationale from superiority to non-inferiority \\
Change in study hypothesis/objective & 4 & 2 & Redefinition of rationale and therapeutic endpoints \\
Total & 59 & 16 &
\end{tabular}

${ }^{\mathrm{a}}$ Based on the registration and results information submission requirements described in Section $80 \mathrm{I}$ of the Food and Drug Administration Amendments Act of 2007 (FDAAA 80I) and the Final Rule for Clinical Trials Registration and Results Information Submission (42 CFR Part II).

${ }^{\mathrm{b}}$ Clearance only valid for FDA scope.

regulatory authorities to have a more cautious stance regarding trials that use such ADs, which reduces their submission clearance rate.

The selected studies include late phase II and III trials covering several therapeutic areas, which are the following: oncology, 21/78 (26.9\%); central nervous system, 15/78 (19.2\%); circulatory system disorders, 13/78 (16.7\%); ophthalmology,
9/78 (11.5\%); infectious diseases, 7/78 (8.9\%); rheumatology, 6/78 (7.7\%); dermatology, 5/78 (6.4\%); and medical devices, 2/78 (2.6\%) (Figure 3).

The vast majority of trials $(53 / 78 ; 68 \%)$ reported industry funding, 15/78 (19\%) reported public healthcare funding, 6/78 (8\%) private foundation funding, and 4/78 (5\%) did not list any funding information. 


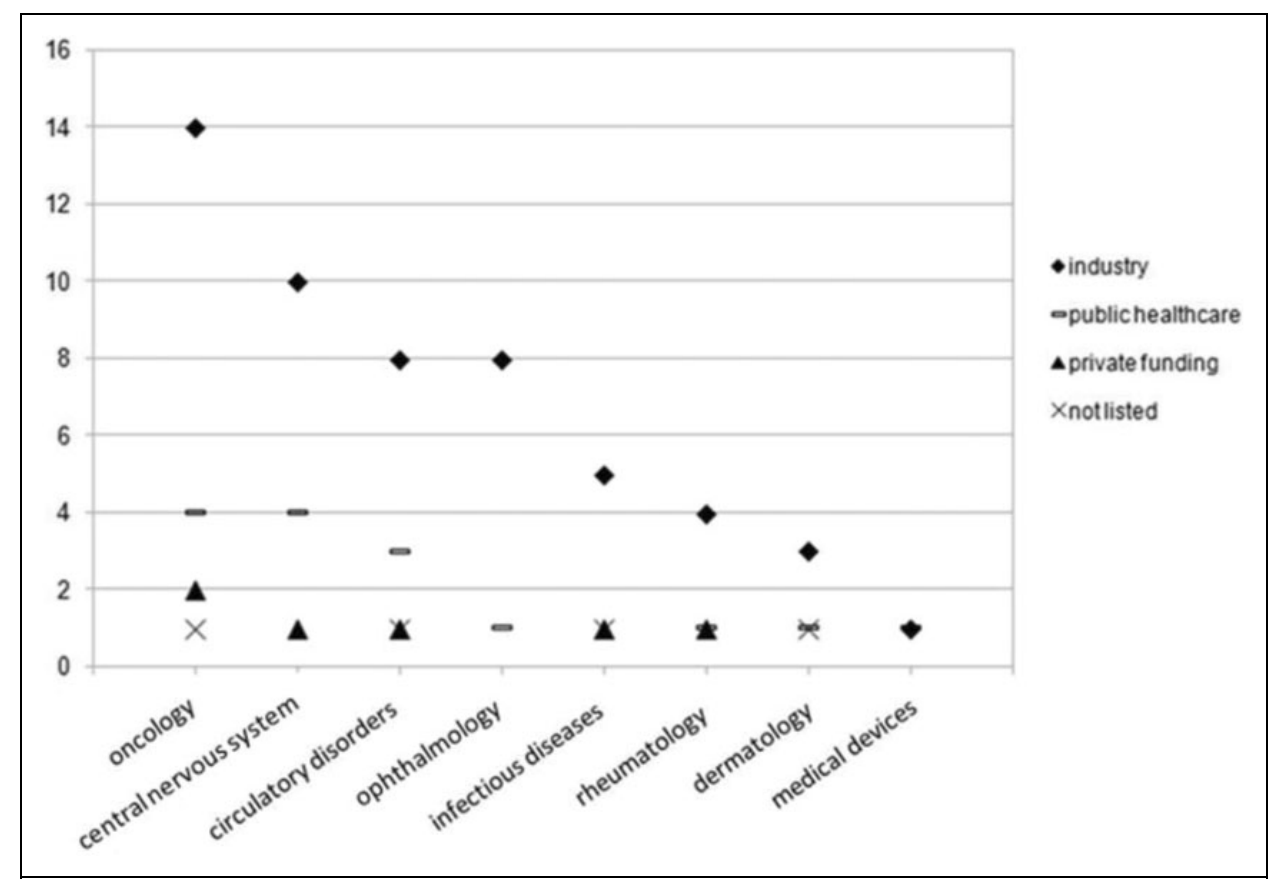

Figure 3. Trials sponsor profile per therapeutic area $(n=78)$.

The sponsor profile of the selected adaptive trials is heterogeneous and often had multiple authors with diverse institutional affiliations. Nearly all trials had at least 1 author affiliated with an academic medical center or hospital 76/78 $(97 \%)$. More than half had at least 1 author affiliated with industry (41/78 (53\%), and 17/78 (22\%) had at least 1 author affiliated with a private investigation center or foundation.

\section{Discussion}

\section{Implementing Adaptive Design in a Clinical Trial}

The various ADs cannot be implemented arbitrarily through all types of CTs since several operational and organizational aspects have to be considered before deciding whether adaptive parameters are applicable and beneficial to the subjects and to the scientific validity of the CT.

Some interim analysis may lead to the suspension of the recruitment process in order to evaluate the interim results and to infer about which $\mathrm{AD}$ to implement. In case of $\mathrm{CT}$, where the duration of treatment is extended or the effect of the therapy is only measurable after a latency period, treatment withdrawal will be more extensive because it is necessary to wait for the results of the last patient included in order to ensure that there is no overflow of the effects of this treatment. ${ }^{10}$

The first step is to choose the appropriate type of AD based on the study objectives. In the case of a confirmatory trial (phase III), a sequential group design or re-estimation of the sample size may be considered. If the timing or number of the interim analyses varies for practical reasons (eg, availability of the data analysis commission [DAC] or safety issues require additional interim analysis) and the estimation of the sample size effect is unreliable, a group sequential design should be used. If the number of doses (study arms) to be considered is greater than 2 (including the control arm), and it is not known exactly which dose (dosage or combination of medication) is most appropriate, the "pick the winner" or "addition of study arm" should be used. ${ }^{10}$

If a stable biomarker evaluation test is available (and is easy to implement) and the experimental drug is expected to have distinct effectiveness and/or safety profiles for subjects with and without biomarker, a biomarker-enriched design can be used in which the interim analysis will be used to select the target population of the study.

In any scenario, a test simulation should be developed for further evaluation and comparison of the operational characteristics of the different designs or parameters of the trial design. For an early-stage trial of a progressive disease, such as cancer, an adaptive dose progression design is used in which the dose is gradually increased according to the progression of the disease so as to preserve patient safety and prevent the risk of subdosing the therapy. ${ }^{11}$

The next step is to determine whether a superiority, noninferiority, or equivalence test is required on the basis of the primary endpoint of the trial and the regulatory requirements, and from that determine the number of interim analysis points. The timing and number of interim analysis will be dependent on safety requirements, statistical efficiency (more interim analysis may reduce sample size), and feasibility (complexity of trial development and associated costs). 
Finally, practical factors have to be considered ${ }^{11}$ :

- How long is the CT?

- Should the trial be suspended during the interim analysis?

- When can the interim analysis and data cleaning be carried out?

- Who will be responsible for carrying out the interim review and drawing up the interim monitoring plan?

- How to perform randomization?

- How will the experimental drug be distributed by the study centers?

- How will the primary analysis of adaptive trial data be performed?

\section{Adaptive Trial Planning}

Given the complexity of adaptive trials and the predetermined nature of any adaptation considered in their conduct, proper planning is imperative. Like any other study, the protocol of a $\mathrm{CT}$ has to be elaborated aiming at the rationale and the primary objectives that it is proposed to achieve. In addition to the statistical challenges that are prioritized, operational aspects also require prior evaluation. For example, timely distribution of experimental drug in the centers should be ensured, although preplanning is hampered by the difficulty in predicting which dose will be maintained or discontinued after interim analysis. ${ }^{12}$

The planning stage also includes the elaboration of the protocol, which is the key document in the structuring of the CT. It is important to note that given the complexity of the methods and additional procedures performed in conducting an $\mathrm{AD}$, this will result in a more extensive and therefore more complex protocol which requires that more elements be included than in a standard test protocol. This includes the justification for the application of an $\mathrm{AD}$ and the advantages resulted from, based on the previous simulation of the trial. In addition, a clear description of the adaptive mechanism should be included, the role of the statistical analysis committee should be explained, and additional discussion related to the control of type I error, its estimation calculations, and error margins should be provided. ${ }^{12}$

In short, the planning stage of a $\mathrm{CT}$ with $\mathrm{AD}$ will be more extensive than in a conventional trial. This is justified by the preplanned nature of the implemented adaptations, and the complexity of this type of study will also imply the allocation of more human and technical resources as multiple tasks will be included in the initial preparation of the trial.

\section{Trial Monitoring}

The extent and nature of the monitoring is determined by the CT sponsor, depending on the type of endpoints and the complexity of the design of the study.

In a $\mathrm{CT}$ with $\mathrm{AD}$, a $\mathrm{DMC}$ is defined that encompasses risk management functions of the trial but also evaluates and safeguards the statistical and scientific integrity of the CT. ${ }^{13}$ It consists of several independent experts assigned to the study by the developer and can have access to unblinded data throughout the trial to be able to formulate recommendations on preplanned adaptations, focusing on the possibility of operational bias. Thus, standard operating procedures should be defined at the beginning of the CT that have the ability to minimize operational bias, that is, standardize procedures that prevent the leakage of unblinded data. ${ }^{14}$

Because of the complexity of ADs, data quality is of great importance to ensure that results are easily interpretable without risk of ambiguity, requiring not only a well-trained research team familiar with increased on-site monitoring but also involves the use of electronic data collection (EDC). EDC provides an opportunity to facilitate the management of results and also speed up the cleaning of study data. ${ }^{14}$

\section{Technical Considerations for Adaptive Design}

The use of AD is very attractive because of its flexibility and effectiveness in identifying clinical benefits of a treatment tested, especially when limited resources and/or time are available. However, before an AD is implemented, the practical aspects of applicability, validity, and robustness have to be checked, which have an impact on the quality of the results and integrity of the study. ${ }^{12}$

Concerning applicability, the following questions arise ${ }^{15}$ :

- Do potential benefits outweigh the additional efforts required to implement $\mathrm{AD}$ ?

- Does the level of difficulty and costs associated with AD justify the gains from its application?

- Does the implementation of AD delay the recruitment of subjects and prolong the duration of the trial?

- How often are the unblinded analysis performed and to whom is the concealment of the results to be broken?

For validity questions, it is reasonable to ask the following questions:

- Can the break of concealment cause bias in the evaluation of treatment effect?

- Can the implementation of $\mathrm{AD}$ affect randomization?

\section{Subjects characteristics}

The selection of study subjects should reflect the total population for which the drug or device may be indicated. This is not the case for studies in the early stages (phase I) when the choice of subjects is influenced by research questions such as human pharmacology. However, for confirmatory trials in later stages (phase III), subjects should faithfully reflect the original patient population. How much the CT subjects represent future users can be influenced by the medical practices and standard treatment level of a particular study center or geographic region. 
The influence of these factors should be reduced and discussed prior to analysis. ${ }^{16}$

\section{Operational bias}

Operational bias is a major source of concern in CT with AD. Generally, in order to minimize it, it is necessary to control and limit access to interim results of groups not blinded during the trial. One way to achieve this is to restrict access by computer coding of such data, which only releases its access at predetermined times in the CT. This should happen if the sponsor wishes to safeguard the possibility of suggesting scientifically valid adaptations in the design of the trial in its course. In addition, depending on the type of AD implemented, it is suggested that the details of the statistical adaptation algorithm be segregated from the protocol procedures in a SAP whose information is forwarded to the data-monitoring committees, ethic committees, and regulatory agencies. This contributes to the scientific integrity of the $\mathrm{CT}$ and reduces the ability of external observers to bias interim results based on the knowledge of adaptations to the protocol. ${ }^{17}$

For a sequential AD, not all investigators involved must be notified that an interim analysis has been performed. An integrated analysis in the background ensures in parallel that the trial follows the defined protocol and minimizes the bias associated with the interim analysis. Similarly, a CT with adaptive selection of a primary endpoint or adaptive change of hypothesis, assuming that all variables are defined according to the established protocol, the decision to change does not need to be communicated to all study centers.

Well-documented control of study development information increases the likelihood that the trial modifications will be scientifically valid, maintain the integrity of the results generated, and their acceptance by regulatory authorities. ${ }^{18}$

\section{Statistical Considerations for Adaptive Design}

From a statistical point of view, significant adaptations or modifications to the CT and/or statistical procedures may (1) result in a deviation in pharmacotherapeutic parameters in the target population, (2) lead to inconsistencies between hypotheses to be tested and their matching statistical tests, and (3) introduce operational bias/variation in data collection.

The sources of bias/variation can be classified into 4 categories $^{19}$ :

- Expectable and controllable, such as changes in laboratory testing procedures and/or diagnostic procedures

- Expectable but not controllable, such as change in study dose and/or duration of treatment

- Not expected but controllable, such as lack of compliance of subjects with the study

- Unexpected and uncontrollable, those are the random errors in observing clinical responses/outcomes

\section{Simulation and modeling of the trial}

As noted above, any adaptation implemented in a CT must be pre-established. Consistent with this premise, the design of the trial is tested prior to the definition of the final design model used.

The CT simulation is a structural approach that mimics all the procedures and methods inherent in conducting a CT and allows determining the influence of the parameters of the test design model used in the results generated. It assists in understanding the impact that any adaptation will produce on the scientific and statistical validity of the CT as well as on its development. $^{20}$

Computer-generated simulations can provide the operational characteristics of the study design in different scenarios. These simulations can evaluate different scenarios with a variable number and timing of interim analysis and can be used to weigh the advantages and disadvantages of different ADs, or an AD compared to a nonadaptive design. They may also provide additional understanding of the required sample size, operational characteristics, and correlate the design of the trial with patient characteristics when it cannot be performed with an analytical method. ${ }^{22}$

Computational simulations have limitations. First, its usefulness and quality are dependent on the ability to design realistic scenarios. Second, simulation programming errors can be difficult to detect, which may lead to choices of test designs that are not appropriate. Third, complex trial designs that involve multiple endpoints or a complicated null hypothesis detection range may be difficult to simulate. ${ }^{21}$

Modeling plays an important role in the characterization of alternative scenarios, such as the process of patient recruitment and abandonment, dose-response profiles, and endpoint correlation. The combination of modeling and simulation is the axis for the evaluation and comparison of alternative designs for the study, including AD, as well as the planning of a specific type of $\mathrm{AD}$ (eg, number and timing of adaptations, impact on rate and power of type I error).

The FDA guidelines for the implementation of AD recognize the importance of CT simulation for establishing the operational characteristics of $\mathrm{AD}$, comparing alternative designs to justify the selection of a specific design and understanding the deductive properties of AD. Therefore, the guidelines attest that adaptive CT simulation notification should be an important component in documentation submitted to the FDA when a sponsor proposes the use of an AD in a developing trial. This document describes in detail the types of design that can be considered in assessments based on simulations of adaptive tests as well as all the elements that should be described when notifying an adaptive $\mathrm{CT}$ simulation. ${ }^{14}$

Given the complexity of the CT with AD, the simulation of the trial is very important to define how each adaptive model will affect the type I error rate, study power, and degree of bias, being required by regulatory agencies that the test information resulting from the $\mathrm{CT}$ simulation be reported on the study protocol. $^{21}$ 


\section{Protocol and statistical analysis plan}

Given the possible existence of sources of operational bias that unhinges the integrity of an adaptive $\mathrm{CT}$, the prospective specifications in the protocol of all aspects of the study design and scheduled analyses are of paramount importance. Thus, an adaptive trial protocol is typically more complex and detailed than in a standard trial. The protocol and associated documentation (such as the simulation report) must contain critical information that allows regulatory agencies to assess the implementation of $\mathrm{AD}$, such as the following ${ }^{12}$ :

- Rationale of the study

- Justification of the design template, including proposed adaptations

- Operational characteristics of the proposed design and type I error rate

- Plan ensuring trial integrity when planning unblinded interim analyses

- Role of AD in CT development strategy

- Objectives of the proposed $\mathrm{AD}$, all possible adjusted adaptations, assumptions, methodology analysis, and quantitative justification of the test design options taken (via simulation)

- Impact of adaptations on the operational characteristics of the trial (eg, type I error rate)

- Summary of models used in planning (eg, disease progression, dropout, dose-response)

- Analytical derivations that demonstrate strict control of type I error rate

- List of professionals involved in the planning and implementation of adaptations and monitoring of the trial

As one of the main features of CTs with AD is the preestablished nature of the adaptations introduced, it is recommended that an autonomous statistical analysis committee be elaborated with an SAP that prospectively includes all the statistical treatment of the planned adaptations that will be detailed in the protocol. ${ }^{12}$

At the end of the drafting of the protocol, the SAP will not only ensure that the statistical procedures resulting from the implemented adaptations are correct and apt to be used during the trial, but also provide an opportunity for the study sponsor to gain regulatory agreement on the adaptive design and associated statistical analysis.

Another aspect of $\mathrm{AD}$ is that the implementation of adaptations occurs without affecting the scientific integrity of the study. An early establishment of an SAP will also contribute to demonstrate that the operational bias, which is noteworthy in unblinded interim analysis, is not relevant as the statistical treatment of the data is predefined prior to the first interim analysis. $^{22}$

Specific elements should include the SAP, which are the following ${ }^{22}$ :
- All adaptations planned prospectively

- Statistical methods that will be used in the implemented adaptations (eg, how to calculate a potential increase in sample size or duration of the test, rule used to select the dose administered)

- Justification for assessing the type I error rate

- Statistical approach to be used to determine therapeutic results

\section{General Limitations of Adaptive Design}

The use of $\mathrm{AD}$ in a $\mathrm{CT}$ for the purpose of modifying its analytical and statistical procedures, based on interim accumulated data, has been practiced for several years in clinical research. Adaptive methodologies are very appealing to clinical investigators for several reasons. First, it reflects clinical practice in an investigational context. Second, it respects ethical paradigms related to the efficacy and safety (toxicity) of the experimental drug under study. Third, it is not only flexible but also efficient in early stages of clinical development. However, questions are raised regarding the reliability of the $P$ values of the parameters determined and their confidence intervals (Table 3 ). In addition, the systematic use of adaptive methodologies can bias the basic rationale of the study, which would make the clinical trial incapable of addressing the primary goals it intends to achieve. ${ }^{23}$

\section{Regulatory limitations}

From the regulatory point of view, the FDA is supportive of $\mathrm{AD}$ although factors defining appropriative implementation are still being worked out. Adaptive trials are reviewed on a case-bycase basis, with caution from sponsors that protocols using innovative approaches are rejected, leading to delays in the approvals. Given the limited experience of the pharmaceutical industry but also regulatory authorities related to adaptive testing, there may be reservations in accepting the scientific validity of such trials. This may be because there are still no universally accepted statistical methods for all possible adaptations. Thus, in certain cases, clear statistical inferences cannot be produced, which makes the regulatory authorities approval process difficult, if not impossible. ${ }^{24}$

The fear that the use of the interim analyses to inform design adjustments may cause bias and put the statistical validity of the $\mathrm{CT}$ at risk is frequent, but this level of anxiety is reduced as the knowledge in the application of $\mathrm{AD}$ accumulates. Thus, concealment procedures should be standardized prior to the start of the trial. ${ }^{24}$

\section{Logistic limitations}

The implementation and conduction of an adaptive trial creates a number of logistical challenges. A robust infrastructure must be established to ensure that the $\mathrm{AD}$ is implemented correctly. All parties involved in the coordination and monitoring of the trial (sponsor, patients, and investigative team as a whole) should have an integrated understanding of the 
Table 3. Challenges and Limitations Associated With Specific Types of Adaptation.

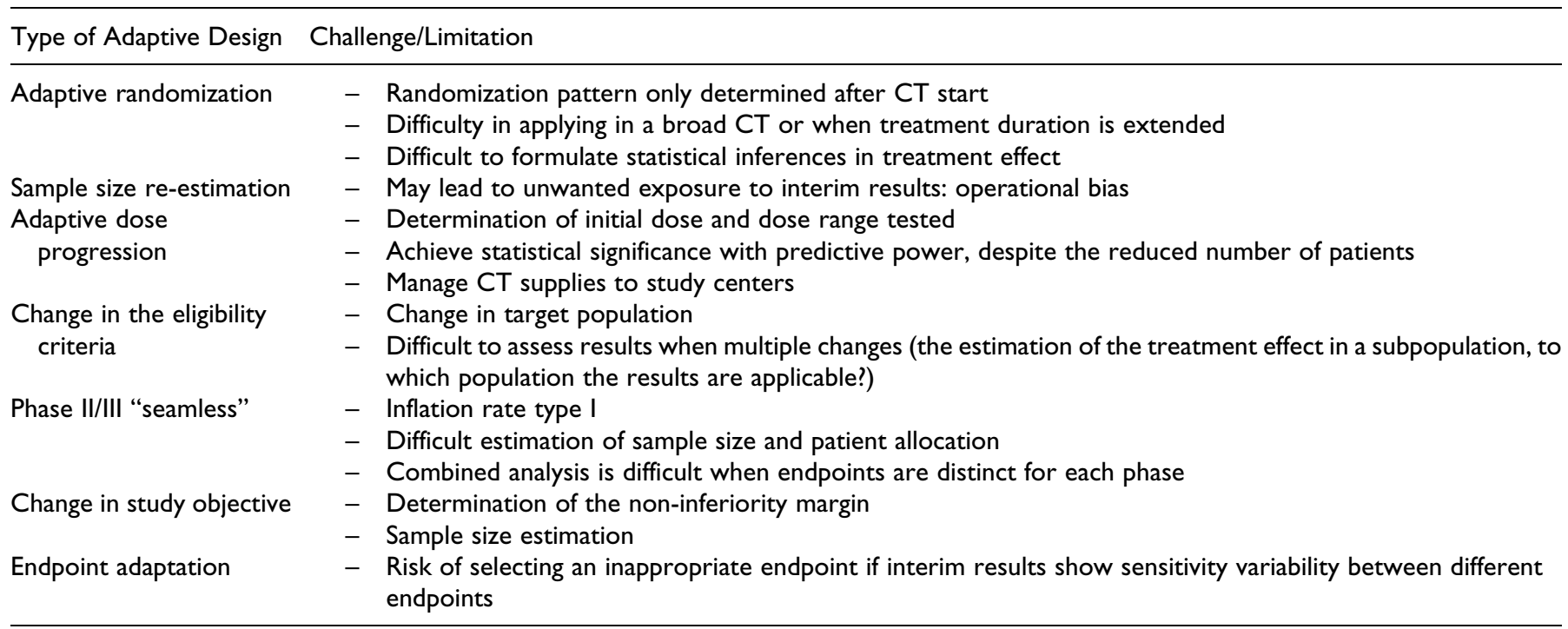

principles of the implemented AD. Interim changes and adaptations in the sample size, objectives, endpoints, or reference values may represent obstacles in the temporal availability of physical resources (physical space that includes all additional procedures), technical (extra equipment required), budget (to cover additional costs to patients in case of extra randomization or treatment allocation procedures), human (if it is necessary to increase the research team because extra procedures are introduced), and statistical tools affecting the quality of the test results. ${ }^{23}$

Various types of adaptations allow treatment arms or doses to be discontinued, more patients to be allocated to a specific treatment arm, or the total sample size recalculated after an interim analysis, making it difficult to prospectively plan the supply of experimental drug to all study centers in adequate amounts. $^{25}$

\section{General Advantages of Adaptive Design}

The FDA's “Clinical Path Opportunities List" lists one of the major advantages of AD with its ability to accelerate the process of developing and approving new drugs. This arises from the possibility of combining distinct phases of clinical investigation or include experimental features in confirmatory trials. The combination of phases in a seamless phase II/III design not only streamlines the conduct of the test (in two distinct phases) but also simplifies the submission and approval process as the two phases are involved in the same submission to the regulatory authorities. ${ }^{27}$ Streamlining the clinical development of a new drug also implies that an effective new treatment can be on the market faster, so it will be accessible to patients sooner. For the pharmaceutical industry, this means that a larger fraction of the patent for a compound is valid when the drug enters the market, which allows a higher return on the investment made.
Another advantage is the flexibility that the AD confers to a CT. For example, if an adaptation is planned prospectively, several characteristics of the study design can be modified based on interim results, which is impossible in a conventional trial frame because of the rigid setting of a conventional design model. ${ }^{26}$

If properly conducted and statistical inference is assured, adaptive trials may also be considered more ethical. The goal of any adaptive trial is to accelerate the clinical development process, which results in fewer patients having to be exposed to an experimental treatment by early discontinuation of an ineffective treatment arm, by changing the inclusion criteria during the trial for those who are more likely to benefit from treatment, reduction of sample size or termination of an early trial for futility, efficacy, or toxicity are included. As already mentioned, this not only implies that fewer patients are exposed to ineffective treatments but also that those treatments that are effective and safe reach the population more quickly. ${ }^{27}$

Overall, the possible benefits of using adaptive design methods in a CT include the following ${ }^{27}$ :

- allows the investigator to correct erroneous assumptions made at the beginning of the CT;

- helps select the most promising therapeutic option during the course of the trial;

- provides the opportunity to react in advance to new pieces of information (positive or negative);

- can shorten the development time of the CT and, consequently, accelerate the overall process of developing the new drug.

\section{Future Perspectives of Adaptive Design in Clinical Trials}

In recent years, there has been an evolution in the orientation of the pharmaceutical industry toward the need to strengthen 
clinical research as well as a general perception that this sector needs to follow the evolution that the activity has experienced recently.

For the pharmaceutical industry, the use of AD will allow more doses to be tested in phases I and II, leading to a better understanding of the effect of the drug on patients at doses that are clinically relevant, leading to better drug development decisions and, for successful drugs, a better phase III trial design, thus helping to reduce the failure of a drug at this stage or, worse, at a later stage of its development. An uninterrupted phase I and phase IIa study will allow efficiency and toxicity to be studied from the start of the CT, with the safety study directly from healthy volunteers. This uniformity will allow the CT to be stopped early if the compound is ineffective or allow it to continue with additional dose arms. ${ }^{28}$

The AD promotes early communication between regulatory agencies and sponsors ensuring more robust study designs. A more constructive collaboration between regulatory agencies, industry, and research centers in the form of consortia will make it more agile, allowing the development of treatment options for pathologies with few therapeutic options, for example, for rare diseases.

For patients, AD will minimize exposure to potentially harmful and ineffective experimental treatments, improve understanding of the disease process and expedite visitation schedules and/or dose regimens.

In the future, it is suggested that the impetus to apply $\mathrm{AD}$ to CT be made with caution, since this methodology is not a "one size fits all," that is, its application is not feasible nor desirable in all the phases of clinical research and in all therapeutic areas. It is advisable to assess the feasibility and the benefits of applying $\mathrm{AD}$ on a case-by-case basis, but also a consideration of the resources (human, budgetary, logistic) necessary for the correct application of this methodology in each study center. ${ }^{29}$

\section{Updated FDA Draft Guidance 2018}

The new adaptive designs draft guidance replaces the earlier draft guidance issued in 2010. The new draft guidance reflects an evolution in FDA's thinking in acknowledging the potential adverse impact arising from type I error and in eliminating confusing categorization of "well understood" and "not well understood" adaptive design methods in favor of a more focused assessment of factors, such as unblinding, that can increase risk of bias.

The draft features discussions on limitations of adaptive trials, how to control the chance for erroneous conclusions, estimating treatment effects, ADs based on comparative data vs noncomparative data and adaptations to the subject population.

Under the special considerations section, the draft also discusses simulations in adaptive design planning, Bayesian adaptive designs, adaptations in time-to-event settings, adaptations based on a potential surrogate or intermediate endpoint, secondary endpoints, adaptive design in early-phase exploratory trials, unplanned design changes based on comparative interim results, and design changes based on information from a source external to the trial. ${ }^{30}$

\section{Conclusions}

When EMA and the FDA published their AD documents in 2007 and 2010, much hope has been placed in these types of CT design to optimize and accelerate the development of new drugs. However, since these adaptive methods are not applicable at all stages and only in specific situations in certain CTs (in terms of sample size, type of endpoints, duration of the CT), their use has only been shown to be advantageous in CTs with an immediate therapeutic effect (allowing the collection of interim efficacy and safety data), with limited number of sites or clinical trials where data cleaning can be easily carried out, which is far short of the pharmaceutical industry investment in this sector. However, many authors suggest that the use of $\mathrm{AD}$ in CTs has several advantages compared with conventional trials.

If properly planned and properly conducted, it can be seen as an opportunity for both the pharmaceutical industry and the regulatory authorities to optimize the overall duration of the development of a medicinal product and allow a faster access of the patient population to new therapeutic solutions.

The Tufts Center for the Study of Drug Development estimates that around $20 \%$ of CTs currently under way are using adaptive designs, while adoption of these techniques is likely to increase significantly over the next few years, particularly in the exploratory phase of drug development. The same study reports that early study terminations due to futility and sample size re-estimation could save sponsor organizations between $\$ 100$ million and $\$ 200$ million annually in aggregate costs. ${ }^{31, \mathrm{i}}$

Adaptive trials should not be viewed as solutions to poorly planned trials. Potential sources of bias should be considered as well as how well the cumulative results of the CT will be properly interpreted and aligned with the primary purpose of the study, the ethical and scientific requirements of the regulatory authorities, and the strategic interests of the sponsor.

Alternatives, whether adaptive or nonadaptive, should be considered, and it should be demonstrated that the selected adaptation has tangible advantages over other options, whether ethical treatment of patients, time and/or resource savings, and better quality of the results and information produced by the CT. The control of the type I error rate (false positive rate) is the main objective of the statistical methodology used (especially in confirmatory tests), so the degree of success will determine the validity of the inferences and results produced.

Logistical problems related to the supply of experimental drugs in different study centers (in the case of administering different doses of the experimental drug), resource allocation, information circulation, and communication may be more challenging constraints than in a conventional clinical trial. The infrastructure of the adaptive design must be arranged in such a way that the flow of information is processed without delays 
that have an impact on the efficiency of the CT. Instructions on efficient data collection, database preparation, decision making, communication and delegation of tasks, and analysis and review of results should be established in advance in order to reinforce the predetermined nature of the $\mathrm{AD}$. 


\section{References}

1. US Food and Drug Administration, Center for Devices and Radiological Health, Center for Biologics Evaluation and Research. Adaptive design clinical trials for drugs and biologics. Draft guidance, 2010. www.fda.gov/downloads/medicaldevices/devicere gulationandguidance/guidancedocuments/ucm446729.pdf. Accessed February 14, 2018.

2. European Medicines Agency. Committee for Medicinal Products for Human Use. Reflection paper on methodological issues in confirmatory clinical trials planned with an adaptive design. London: EMEA; 2007.

3. Park JJ, Thorlund K, Mills EJ. Critical concepts in adaptive clinical trials. Clin Epidemiol. 2018;10:343-351.

4. Chow SC, Chang M. Adaptive design methods in clinical trialsa review. Orphanet J Rare Dis. 2008;3(1):11.

5. Chow SC, Liu JP. Design and Analysis of Clinical Trials: Concepts and Methodologies. 3rd ed. Hoboken, NJ: John Wiley \& Sons Inc; 2014.

6. Bhatt DL, Mehta C. Adaptive designs for clinical trials. $N$ Engl J Med. 2016;375(1):65-74.

7. Chow SC. Adaptive clinical trial design. Annu Rev Med. 2014;65: 405-415.

8. Elsäßer A, Regnstrom J, Vetter T, et al. Adaptive clinical trial designs for European marketing authorization: a survey of scientific advice letters from the European Medicines Agency. Trials. 2014;15(1):383.

9. Brannath W, Burger HU, Glimm E, Stallard N, Vandemeulebroecke $\mathrm{M}$, Wassmer G. Comments on the draft guidance on "adaptive design clinical trials for drugs and biologics" of the US Food and Drug Administration." J Biopharm Stat. 2010; 20(6):1125-1131.

10. Quinlan A, Krams M. Implementing adaptive designs: logistical and operational considerations. Therapeutic Innovation \& Regulatory Science. 2006;40(4):437-444

11. Wang SJ.Regulatory experience of adaptive designs in wellcontrolled clinical trials. Paper presented at: Adaptive Designs: Opportunities, Challenges and Scope in Drug Development, Washington, DC, 2006.

12. Gallo P, Maurer W. Challenges to implementing adaptive designs: comments on the viewpoints expressed by regulatory biostatisticians. Biom J. 2006;48(4):591-597.

13. Baber N. International Conference on Harmonization of Technical Requirements for Registration of Pharmaceuticals for Human Use (ICH). Br J Clin Pharmacol. 1994;37(5):401-404.

14. Tsiatis A, Mehta C. On the inefficiency of the adaptive design for monitoring clinical trials. Biometrika. 2003;90(2):367-378.

15. Hu F, Rosenberger WF. Optimality, variability, power: evaluating response-adaptive randomization procedures for treatment comparisons. J Am Stat Assoc. 2003;98:671-678.

16. Berry DA. Adaptive clinical trials in oncology. Nat Rev Clin Oncol. 2011;9(4):199-207.

17. Lavori PW, Dawson R. A design for testing clinical strategies: biased adaptive within-subject randomization. J R Stat Soc. 2000; 163(1):29-38.

18. Tsiatis AA. Information based monitoring of clinical trials. Stat Med. 2006;25:3236-3244.

19. Kieser M, Friede T. Re-calculating the sample size in internal pilot study designs with control of the type I error rate. Stat Med. 2000;19:901-911.
20. Li HI, Lai PY. Clinical trial simulation. In: Chow SC, ed. Encyclopedia of Biofarmaceutical Statistics. New York, NY: Marcel Dekker, Inc; 2003:200-201.

21. Holford NHG, Kimko HC, Monteleone JPR, et al. Simulation of clinical trials. Annu Rev Pharmacol Toxicol. 2000;40: 209-234.

22. Hung HMJ, Wang SJ, O’Neill R. Statistical considerations for testing multiple endpoints in group sequential or adaptive clinical trials. J Biopharm Stat. 2007;17(6):1201-1210.

23. Triphaty V. Adaptive clinical trials: challenges and opportunities. www.tcs.com/SiteCollectionDocuments/White\%20Papers/LSWhitePaper-Adaptive-clinical-trials-challenges-opportunites0713\%20-1.pdf. Published 2013. Accessed January 22, 2018.

24. Hung HM, O'Neill RT, Wang SJ, Lawrence J. A regulatory view on adaptive/flexible clinical trial design. Biom J. 2006;48(4): 565-573.

25. Chow SC, Shao J. Inference for clinical trials with some protocol amendments. J Biopharm Stat. 2005;15:659-666.

26. Woodcock J, Woosley R. The FDA critical path initiative and its influence on new drug development. Annu Rev Med. 2008;59:1-12.

27. Müller $H H$, Schäfer H. Adaptive group sequential designs for clinical trials: combining the advantages of adaptive and of classical group sequential approaches. Biometrics. 2001;57(3): 886-891.

28. Gallo P, Chuang-Stein C, Dragalin V, Gaydos B, Krams M, Pinheiro J; PhRMA Working Group. Adaptive designs in clinical drug development - an executive summary of the PhRMA working group. J Biopharm Stat. 2006;16(3):275-283.

29. Mahajan R, Gupta K. Adaptive design clinical trials: methodology, challenges and prospect. Indian J Pharmacol. 2010;42(4):201-207.

30. US Department of Health and Human Services, US Food and Drug Administration, Center for Drug Evaluation and Research, Center for Biologics Evaluation and Research. Adaptive design clinical trials for drugs and biologics. New Updated Draft Guidance, 2018. https://www.fda.gov/downloads/drugs/guidances/ ucm201790.pdf. Accessed November 1, 2018.

31. R\&D. Senior Leadership Brief. The adoption and impact of adaptive trial designs. Tufts Center for the Study of Drug Development, Tufts University, 2013. https://static1.squarespace.com/ static/5a9eb0c8e2ccd1158288d8dc/t/5aa300cd0d9297d1cce 5515a/1520632014953/tuftscsddbrief1 final_new.pdf. Accessed November 11, 2018. 